\title{
Isotope effect on the detachment onset density in JET ohmic plasmas
}

\author{
V Solokha ${ }^{1}, \mathrm{M}$ Groth $^{1}, \mathrm{~S}$ Brezinsek $^{2}, \mathrm{M}$ Brix $^{3}$, \\ G Corrigan ${ }^{3}$, C Guillemaut ${ }^{4}$, D Harting ${ }^{3}$, S Jachmich ${ }^{5}$, \\ $\mathrm{U} K r u e z i^{6}$, S Marsen ${ }^{7}$, S Wiesen ${ }^{2}$, and JET contributors* \\ 1 Aalto University, P.O. Box 14100, FI-00076, Aalto, Espoo, Finland \\ 2 Forschungszentrum Julich GmbH, Institut fur Energie- und Klimaforschung, \\ IEK-4 - Plasmaphysik, 52425 Julich, Germany \\ ${ }^{3}$ EUROfusion Consortium, JET, Culham Science Centre, Abingdon, OX14 \\ 3DB, UK \\ ${ }^{4}$ Instituto de Plasmas e Fusao Nuclear, Instituto Superior Tecnico, Lisbon, \\ Portugal \\ 5 Ecole Royale Militaire School, Av de la Renaissance 30, Brussels, Belgium \\ ${ }^{6}$ ITER Organization Route de Vinon sur Verdon, Saint Paul-lez-Durance, \\ France \\ 7 Max-Planck-Institute for Plasma Physics, Greifswald, Germany \\ * See the author list of "Overview of the JET preparation for \\ Deuterium-Tritium Operation" by E. Joffrin et al. to be published in Nuclear \\ Fusion Special issue: overview and summary reports from the 27th Fusion \\ Energy Conference (Ahmedabad, India, 22-27 October 2018) \\ E-mail: vladimir.solokha@aalto.fi
}

May 2019

\begin{abstract}
This paper investigates the dependence of the detachment onset density on the hydrogen isotope species. In JET ohmic plasmas in the ITERlike wall (JET-ILW) materials configuration, the isotope effect is approximately $10 \%$, and it was observed at the outer target only. The heavier isotopes exhibit lower detachment onset densities and density limits. In addition, higher subdivertor molecular pressures and divertor electron densities were observed in the deuterium (D) discharges compared to the hydrogen $(\mathrm{H})$ discharges.

EDGE2D-EIRENE and standalone EIRENE simulations show that lower conductance of the pumping plenum, scaling with $\sqrt{m_{D} / m_{H}}$ in the molecular flow regime present in the experiments, is the primary cause for the experimental observation.
\end{abstract}

\section{Introduction}

The goal of ITER is to demonstrate the technological feasibility of fusion power. In the ITER baseline scenario, approximately $100 \mathrm{MW}$ of fusion power is expected to cross the separatrix, enter the scrape-off layer (SOL), which results in divertor heat fluxes in excess of $10 \mathrm{MW} / \mathrm{m}^{2}$ deposited onto the target plates $[1,2,3]$.

Such high heat fluxes are reduced by operating in the detached regime. Experimentally, the detached regime is characterized by the decrease of the ion current to inner (IT) and outer (OT) targets. In the detached regime, volumetric recombination currents become comparable, or even higher, than the ionization 


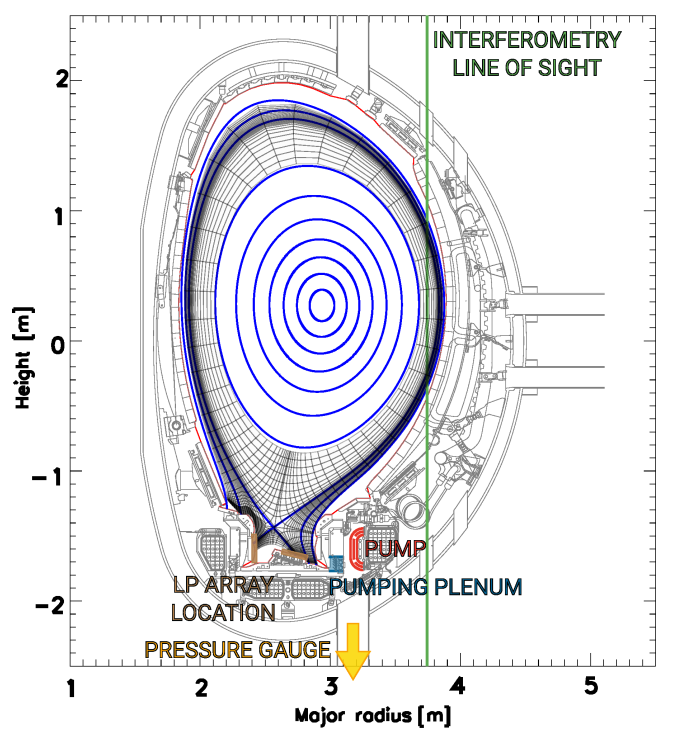

Figure 1. The magnetic configuration of JET Pulse Number 80295 (blue), interferometry line of sight (green), cryopump (red), location of the Langmuir probes array (brown), pressure gauge (yellow), and pumping plenum (cyan). The pressure gauge is mounted at the end of vertical port approximately $3 \mathrm{~m}$ below the subdivertor structure. The EDGE2D computational grid is overlaid in black.

currents, which take place at plasma temperatures below a few $\mathrm{eV}$ and plasma densities above $10^{20} \mathrm{~m}^{-3}$. These conditions causes a rapid increase in atomic and molecular densities at targets and a decrease of particle and heat loads to the divertor targets due to momentum and power losses caused by a ion-neutral friction and a radiation [4].

Previous studies by Maggi et al. [5] of the isotope effect in JET with carbon-based plasma facing components (JET-C) for a vertical target plasma configuration showed that both the detachment onset density and the density limit scale inversely with the main ion mass. For these plasmas, D plasmas had a 15-20\% lower detachment onset density than in otherwise identical $\mathrm{H}$ plasmas.

There are several physics processes which depend on the hydrogen isotope species: the ionisation mean free path (MFP), the Bohm condition at the sheath, and the pumping plenum conductance. The impact of the main ion masses on the detachment onset density was experimental and numerically assessed for the JET ITER-like wall (JET-ILW) materials configuration in plasma configurations with the high-field strike point on the vertical target plate, and the low-field strike point on the horizontal plate (Fig. 1). The experimental studies include $\mathrm{H}$ and $\mathrm{D}$ fuelling ramps to the density limit as well as density steps with strike point sweeps for target Langmuir probe measurements. Simulations with the coupled fluid edge/neutral Monte-Carlo code EDGE2D-EIRENE $[6,7]$ and standalone EIRENE $[8,9]$ were carried out to replicate the measured plasma conditions in the divertor, and to assess neutral transport in the divertor as well as across the subdivertor structure to the cryopump. The present studies extend those of the Uljanovs et al. [10] with more detailed investigation of the impact of the pumping plenum. 


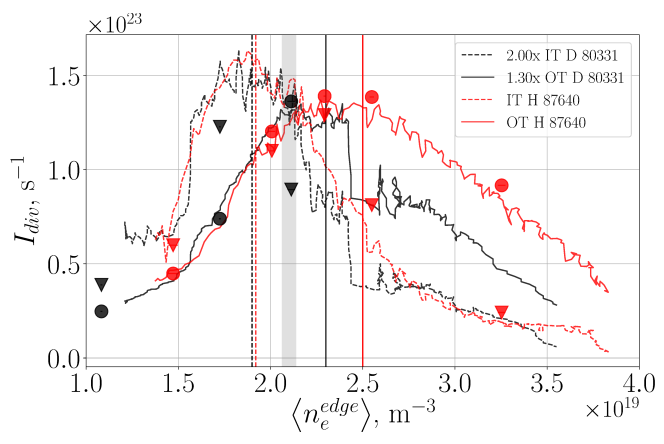

Figure 2. Total ion current to divertor inner targets (solid lines and triangle markers) and outer targets (dashed lines and circle markers) in $\mathrm{H}$ discharges (red) and D discharges (black). The deuterium data were scaled to match $\mathrm{H}$ case values for visualisation purpose only. The data were used from two ohmic JET discharges with linearly increased edge density (lines): JPN 80331 (D) and JPN $87640(\mathrm{H})$; and six density plateau discharges OT (circles) and IT (triangles): JPN 80326 (D), JPN 80327 (D), JPN 80328 (D), JPN 80329 (D), JPN 87788 (H) and JPN $87795(\mathrm{H})$.

\section{Experimental results}

Fuelling ramp and discrete density steps were performed in JET ohmic discharges with $I_{p}=2 \mathrm{MA}, B_{T}=2 \mathrm{~T}$ and $P_{O H}=2 \mathrm{MW}$. The total ion current to the $\operatorname{IT}\left(I_{\text {div }}^{I T}\right)$ and OT $\left(I_{\text {div }}^{I T}\right)$ as measured by Langmuir probes increased along with line-averaged density measured in the edge region of the main plasma $\left\langle n_{e}^{\text {edge }}\right\rangle$ (Fig.1), in the sheathlimited and the high-recycling regimes (Fig.2). At edge densities of $1.9 \cdot 10^{19} \mathrm{~m}^{-3}$ and $2.4 \cdot 10^{19} \mathrm{~m}^{-3}$ the total ion current to the IT and the OT, respectively, rolled over indicating the onset of detachment. There is no significant difference between the ion currents measured in fuelling ramps and density steps. At the OT the rollover density is $10 \%$ higher in $\mathrm{H}$ discharges than in $\mathrm{D}$ discharges.

The molecular pressure in the subdivertor was 30-40\% higher for D discharges at moderate densities $\left(n_{e, \text { sep }}^{u p}=10^{19} \mathrm{~m}^{-3}\right.$ ) compared to $\mathrm{H}$ (Fig.3). Here, the measurements from the fuelling ramps were omitted, and data shown for the density steps only, as the pressure equilibration between the divertor and the position of the pressure gauge typically takes $200 \mathrm{~ms}$. The comparison between the experimental subdivertor pressures (in density plateau discharges) and EDGE2DEIRENE simulations results was done using the $n_{e, \text { sep }}^{u p} \propto\left\langle n_{e}^{\text {edge }}\right\rangle$ scaling postulated by Groth et al [11].

The electron density across the outer target plate in the D case is $\sim 20 \%$ higher and the electron temperature is lower than in the $\mathrm{H}$ case (Fig.4). One possible cause for this observation is that the higher neutral densities in $\mathrm{D}$ discharges than in the $\mathrm{H}$ discharges, supported by higher subdivertor pressure measured for $\mathrm{D}$ than for $\mathrm{H}$.

\section{Numerical simulations}

The setup of EDGE2D-EIRENE, including the choice of ad-hoc cross-field particle diffusion and thermal conductivity coefficients, and the inclusion of the subdivertor, were identical to Ref. [10] and diffusion coefficient were equal to $0.5 \mathrm{~m}^{2} / \mathrm{s}$ near the separatrix and $1.0 \mathrm{~m}^{2} / \mathrm{s}$ in the distance more than $2 \mathrm{~cm}$ from separatrix, thermal 


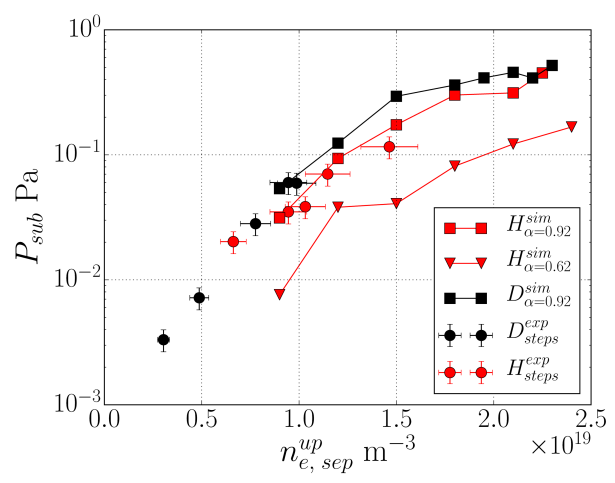

Figure 3. The subdiveror pressure measurements (log scale) for the density step discharges (circles) compared with the EDGE2D-EIRENE simulations (symbols with lines). The x-axis is upstream density (electron density at the midplane separatrix as obtained in [11]).

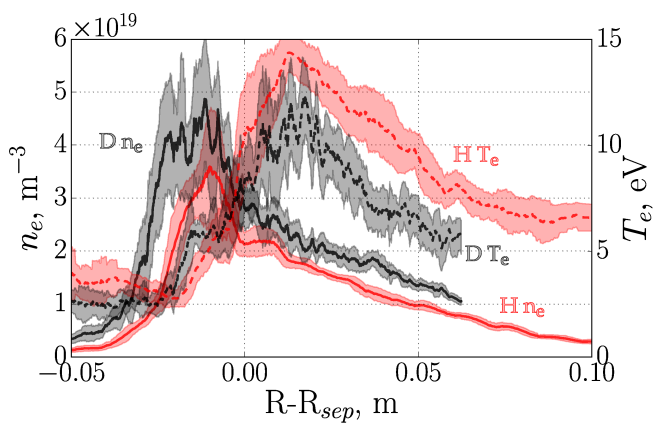

Figure 4. Langmuir probe $n_{e}$ (solid) and $T_{e}$ (dashed) measurements at $\mathrm{OT}$ in JPN $87795 \mathrm{H}$ (red) and JPN $80328 \mathrm{D}$ (black) discharges at $\left\langle n_{e}^{\text {edge }}\right\rangle=1.7 \cdot 10^{19}$ $\mathrm{m}^{-3}$.

conductivity coefficient had a step from $1.0 \mathrm{~m}^{2} / \mathrm{s}$ to $0.5 \mathrm{~m}^{2} / \mathrm{s}$ at the separatrix. The same setup is used for both isotopes. Cross-field drifts are omitted in this publication.

Consistent with the measured currents, EDGE2D-EIRENE simulations showed that the current rollover occurs at almost the same density for $\mathrm{H}$ simulations and for $\mathrm{D}$ simulations. The detachment is less pronounced in the EDGE2D-EIRENE simulations than in the experiment (Fig.5).

The measured and predicted subdivertor pressures can be matched in the cryopump albedo range from $\alpha=0.62$ to $\alpha=0.92$ (Fig.3). Previous EDGE2DEIRENE simulations for L-mode plasmas assumed an cryopump albedo of 0.92 to match the deuterium subdivertor pressure and the deuterium current [12]. In these ohmic plasmas, an albedo of 0.88 was found more appropriate, which is within the uncertainty of the measurements.

The EIRENE predicted OT atomic and molecular densities in the D case were $10 \%$ and $40 \%$ higher than in $\mathrm{H}$ case, respectively (Fig.6).

To investigate the cause of the difference of the molecular content in the divertor we performed standalone EIRENE simulations in vacuum conditions in which the 


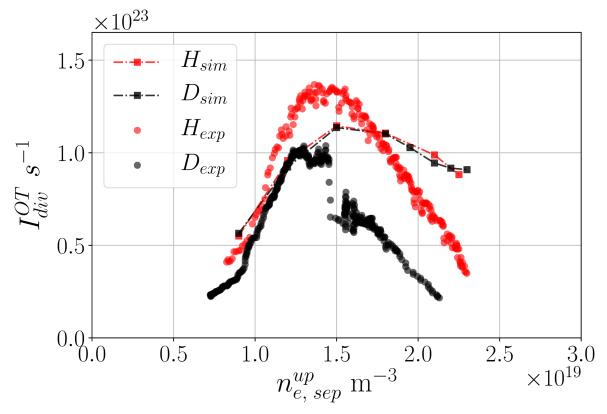

Figure 5. Total outer target ion current as a function of upstream density in experiments (symbols) and in EDGE2D-EIRENE simulations (symbols with lines) density scans in $\mathrm{H}$ (red) and $\mathrm{D}$ (black).

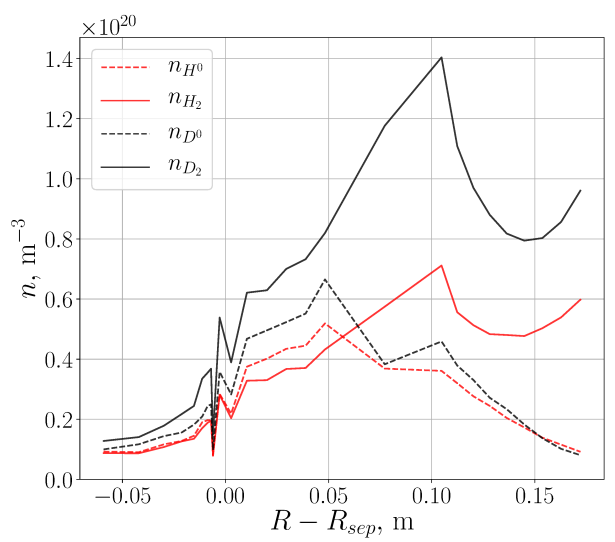

Figure 6. Divertor OT molecular (soild lines) and atomic (dashed lines) density in EDGE2D-EIRENE simulations at $n_{e, \text { sep }}^{u p}=1.8 \cdot 10^{19} \mathrm{~m}^{-3}$ for $\mathrm{H}$ (red) and D (black).

molecule source $\left(I=1.9 \cdot 10^{23} \mathrm{~s}^{-1}\right)$ was located at the top of the vacuum vessel. The absence of the plasma allows for separating the impact of the divertor and strike point geometry on the trajectories of the molecules from plasma effects, such as ionisation mean free path. The cryopump albedo for both isotopes was assumed equal to $\alpha=0.92$. The molecular pressure of the ohmic discharges is sufficiently low $\left(p_{\text {sub }}<0.1 \mathrm{~Pa}\right)$ to neglect the influence of neutral-neutral collisions.

The EIRENE simulations showed that in molecular flow conditions $n_{D_{2}} / n_{H_{2}}=$ 1.41 is valid for the entire device. This ratio is a consequence of difference in subdivertor entrance conductances. The conductance in the molecular flow regime depends on the thermal velocity $\left(v^{T} \sim \sqrt{T / m}\right)$, thus the pumping plenum conductance is lower for heavier molecules. In the limit of the equal fluxes, the density in the $\mathrm{D}$ case is $v_{H}^{T} / v_{D}^{T} \sim \sqrt{m_{D} / m_{H}}$ times higher.

The higher atomic and molecular densities increase the power losses caused by a radiation in the divertor region at rollover densities $\left(n_{e, \text { sep }}^{\text {up }}=1.8 \cdot 10^{19} \mathrm{~m}^{-3}\right)$ by $\mathrm{a} \sim 10 \%$ and atoms ionisation power losses by a $\sim 20 \%$ according to the EDGE2D- 


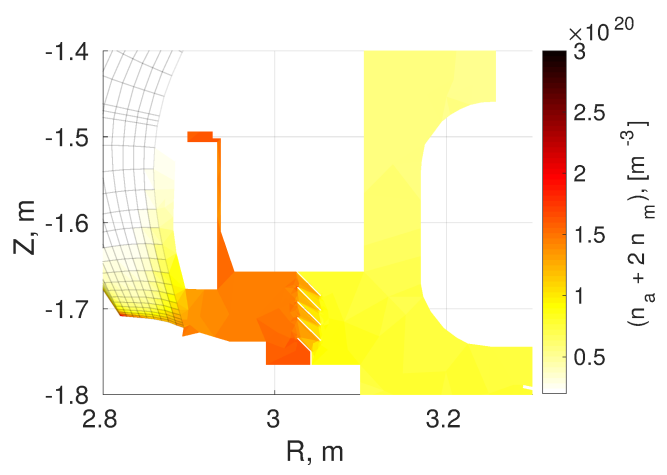

Figure 7. The neutral particle $\left(n_{a}+2 n_{m}\right)$ density in the EDGE2D-EIRENE simulation of high-recycling $\left(n_{e, \text { sep }}^{u p}=1.5 \cdot 10^{19} \mathrm{~m}^{-3}\right) D$ discharge with louvre included.

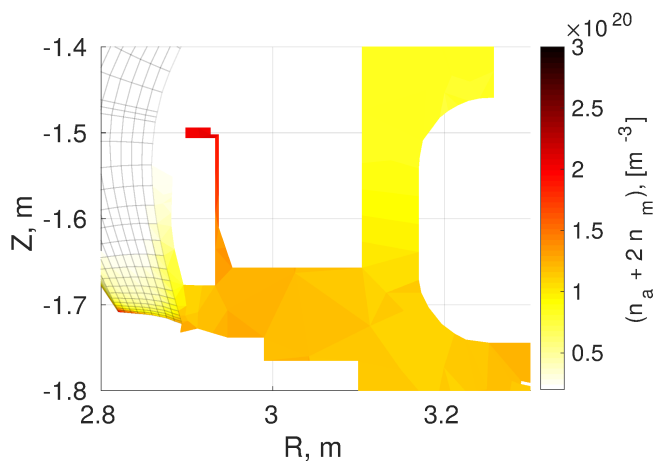

Figure 8. The neutral particle $\left(n_{a}+2 n_{m}\right)$ density in the EDGE2D-EIRENE simulation of high-recycling $\left(n_{e}^{u p}\right.$, sep $\left.=1.5 \cdot 10^{19} \mathrm{~m}^{-3}\right) D$ discharge without louvre.

EIRENE simulations. It is one of the causes of the lower rollover density in the D cases.

The simulations also showed that the louvre will reflect $80 \%$ of particles back to the divertor. The reflection reduces the probability of the molecules to reach the cryopump. Thus the cryopump ability to pump D more efficiently than the $\mathrm{H}$ [13] would not play a role.

Effectively, the louvre creates a high density of molecules on the divertor side of the louvres (Fig.7, 8). It makes detachment onset density lower for both isotopes.

Standalone EIRENE simulations of particle sources at the IT and OT strike points with a static background plasma $\left(n_{e, \text { sep }}^{u p}=1.5 \cdot 10^{19} \mathrm{~m}^{-3}\right)$ showed that probability for recycled particles to enter the OT pumping plenum rather than IT pumping plenum is $\sim 2$ times higher despite the higher total ion currents at the IT. The asymmetry is caused by the specific geometry of the JET-ILW divertor and strike point positions investigated in these studies. The higher probability of reaching the OT pumping plenum is favourable for pumping, but it makes the isotope effect occur mostly at the OT. 


\section{Conclusions}

The subdivertor pumping plenum conductance depends linearly on the thermal velocity of molecules, which is predicted to cause higher neutral densities in deuterium than in hydrogen plasmas for JET-ILW discharges with the outer strike point on the horizontal target plate. The relative difference in densities is proportional to the square root of the isotope mass ratio in the case of the equal fuelling current.

In the experiments, the detachment onset density at the OT in D cases is $10 \%$ lower than in $\mathrm{H}$ cases. A 20-30\% higher subdivertor molecular pressure and divertor electron densities in $\mathrm{D}$ were observed. Our studies show that these difference could be caused by the different pumping plenum conductivity leading higher molecular densities in the divertor for heavier isotopes. The IT is separated by the divertor plasma from the OT pumping plenum, thus it cannot be pumped as efficient as the OT region. Therefore, the difference in the pumping plenum conductivity will not affect the molecular densities near the IT. It explains the absence of the isotope effect at the IT. The difference in the MFP of isotopes also plays a role, but the typical MFP length is less than $5 \mathrm{~cm}$ in the JET-ILW high-recycling conditions, and it varies along the OT by a several orders of maginitude. The impact of the MFP on the detachment was out of scope of this paper.

The louvre reduces the efficiency of pumping and creates a high molecular density near the outer target. It decreases the detachment onset density for both isotopes.

EDGE2D-EIRENE simulations showed the divertor molecules content and the OT molecular density in the D case is $30 \%$ higher than in the $\mathrm{H}$ case in sheathlimited and high-recycling regimes. It is presumably caused by the pumping plenum conductance dependency on the hydrogen isotope species. This could increase the volumetric recombination near the target due to the molecular assisted recombination and power dissipation. The conclusions are valid for the neutral pressure below the $0.1 \mathrm{~Pa}$ in the subdivertor, which justifies the approximation of molecular flow in the pumping plenum. This assumption is only valid for ohmic discharges at JET.

\section{Acknowledgement}

This work has been carried out within the framework of the EUROfusion Consortium and has received funding from the Euratom research and training programme 20142018 and 2019-2020 under grant agreement No 633053. The views and opinions expressed herein do not necessarily reflect those of the European Commission.

\section{References}

[1] C.S. Chang et al. Nucl. Fusion, 57 (2017), 116023.

[2] X. Xu et al. IAEA FEC (2018), IAEA-CN-123/45/TH/P7-21.

[3] ITER Physics Expert Group et al. Nucl. Fusion, 39 (1999), 2391.

[4] S.I. Krasheninnikov, A. S. Kukushkin, and A. A. Pshenov Phys. Plasmas 23 (2016), 055602

[5] C.F. Maggi et al. Nucl. Fusion, 39 (1999), 979.

[6] R. Simonini et al, Contrib. Plasma Physics, 34 (1994), 368-373.

[7] S. Wiesen, JET ITC-Report, (2006), http://www.eirene.de/e2deir_report_30jun06.pdf

[8] D. Reiter, Julich KFA Report (2005), http://www.eirene.de

[9] D. Reiter, M. Baelmans and P. Borner, Fusion Sci. Technol. (2005), 47:2, 172-186

[10] J. Uljanovs et al, Nuclear Materials and Energy, 12 (2017), 791-797

[11] M. Groth, J. Nucl. Mater., 438 (2013), S175S179

[12] D. Moulton et al, $42^{\text {nd }}$ EPS Conference on Plasma Physics, (2015), O4.119. 
[13] C. Day, CAS - CERN Accelerator School and ALBA Synchrotron Light Facility: Course on Vacuum in Accelerators, Platja d'Aro, Spain, 16 - 24 May 2006, pp.241-274 